\title{
Cigarette smoke and HIV synergistically affect lung pathology in cynomolgus macaques
}

\author{
Hitendra S. Chand, ${ }^{1,2}$ Rodrigo Vazquez-Guillamet, ${ }^{3}$ Christopher Royer, ${ }^{1}$ Karin Rudolph, ${ }^{1}$ Neerad Mishra, ${ }^{1}$ Shashi P. Singh, ${ }^{1}$ \\ Shah S. Hussain, ${ }^{2}$ Edward Barrett, ${ }^{1}$ Shannon Callen, ${ }^{4}$ Siddappa N. Byrareddy, ${ }^{4}$ Maria Cristina Vazquez Guillamet, ${ }^{3}$ \\ Jawad Abukhalaf,, ${ }^{3}$ Aryaz Sheybani, ${ }^{3}$ Vernat Exil, ${ }^{3}$ Veena Raizada, ${ }^{3}$ Hemant Agarwal, ${ }^{3}$ Madhavan Nair, ${ }^{2}$ \\ Francois Villinger, ${ }^{5}$ Shilpa Buch, ${ }^{4}$ and Mohan Sopori ${ }^{1}$ \\ 'Lovelace Respiratory Research Institute, Albuquerque, New Mexico, USA. ${ }^{2}$ Herbert Wertheim College of Medicine, Florida International University, Miami, Florida, USA. ${ }^{3}$ University of New Mexico Health \\ Sciences Center, Albuquerque, New Mexico, USA. ${ }^{4}$ University of Nebraska Medical Center, Omaha, Nebraska, USA. ${ }^{5}$ New Iberia Research Center, University of Louisiana at Lafayette, New Iberia, Louisiana, USA.
}

\begin{abstract}
In the era of combined antiretroviral therapy (cART), lung diseases such as chronic bronchitis (CB) and chronic obstructive pulmonary disease (COPD) are common among persons living with HIV (PLWH), particularly smokers. Although smoking is highly prevalent among PLWH, HIV may be an independent risk factor for lung diseases; however, the role of HIV and cigarette smoke (CS) and their potential interaction in the development of chronic lung diseases among PLWH has not been delineated. To investigate this interaction, cynomolgus macaques were exposed to CS and/or simian-adapted human immunodeficiency virus (SHIV) and treated with CART. The development of CB and the lung functions were evaluated following CS \pm SHIV treatment. The results showed that in the lung, SHIV was a strong independent risk factor for goblet cell metaplasia/hyperplasia and mucus formation, MUC5AC synthesis, loss of tight junction proteins, and increased expression of Th2 cytokines/transcription factors. In addition, SHIV and CS synergistically reduced lung function and increased extrathoracic tracheal ring thickness. Interestingly, SHIV infection generated significant numbers of HIV-gp120+ epithelial cells (HGECs) in small airways and alveoli, and their numbers doubled in CS+SHIV-infected lungs. We conclude that even with CART, SHIV independently induces CB and pro-COPD changes in the lung, and the effects are exacerbated by CS.
\end{abstract}

\section{Introduction}

Although cART has dramatically improved the life span of persons living with HIV (PLWH) and transformed the infection to more or less a medically manageable chronic disease (1), the incidence of chronic lung diseases including chronic bronchitis (CB) and chronic obstructive pulmonary disease (COPD) is high in PLWH $(2,3)$. While the incidence of asthma among HIV-seropositive children is high (4), the frequency of asthma in adult PLWH is debatable $(5,6)$. Generally, noninfectious chronic lung diseases are associated/affected by CS $(7,8)$, and smoking is highly prevalent among PLWH $(9,10)$. Epidemiological data suggest HIV as an independent risk factor for COPD $(11,12)$ and CS and lung infections synergize to promote COPD (13). Thus, interaction between CS and HIV may affect development of obstructive lung diseases in PLWH who are smokers.

$\mathrm{CB}$ or chronic mucus hypersecretion $(\mathrm{CMH})(14)$ results from mucus overproduction by goblet cells, leading to airway obstruction and decline in lung function $(15,16)$. COPD encompasses a spectrum of diseases including $\mathrm{CB}$, reduced lung function, and emphysema and its severity is linked to $\mathrm{CMH}$ (17). We have shown that, even after well-controlled viremia, SIV- and HIV-infected lungs contain large numbers of mucous ${ }^{+}$gp $120^{+}$-immunoreactive

Conflict of interest: The authors have declared that no conflict of interest exists. License: Copyright 2018, American Society for Clinical Investigation.

Submitted: May 1, 2018; Accepted: September 27, 2018.

Reference information: J Clin Invest. 2018;128(12):5428-5433.

https://doi.org/10.1172/JCl121935. cells (18). Moreover, exposure of normal human bronchial epithelial (NHBE) cells to approximately $8 \times 10^{-11} \mathrm{M}$ HIV-gp120 induces CXCR4- and nicotinic acetylcholine receptor-dependent mucus production and goblet cell hyperplasia (18), suggesting a potential interaction between CS and HIV in CB development. Furthermore, HIV may infect NHBE cells either unproductively (19) or productively (20). Therefore, we investigated the interaction between CS and SHIV infection in the development of lung diseases in cynomolgus macaques.

\section{Results and Discussion}

Cynomolgus macaques were exposed to CS for 27 weeks and/ or SHIV infection for 16 weeks to obtain 4 experimental groups: fresh air control (FA), CS-exposed, SHIV-infected, and CS+SHIV (see Supplemental Methods and Supplemental Figure 1; supplemental material available online with this article; https:// doi.org/10.1172/JCI121935DS1). All SHIV-infected macaques received daily cART starting at 2 weeks postinfection until sacrifice. To determine the nature of lung cells that are affected by CS and SHIV, we enumerated HIV-gp $120^{+}$and $\mathrm{pCK}^{+}$(epithelial cell marker) double-positive cells (i.e., HGECs) in the small airways and alveoli. While SHIV-infected animals had 9.0\% $\pm 1.1 \%$ and $15.8 \% \pm 1.6 \%$ HGECs in the airways (Figure $1 \mathrm{~A}$ ) and in alveoli (Figure 1B), respectively, CS nearly doubled the number in both airways and alveoli to $19.0 \% \pm 2.2 \%$ and $29.6 \% \pm 2.6 \%$, respectively (Figure 1, A and B). Plasma viral titers were measured at 2 weeks postinfection to analyze the effects of CS on viral replica- 
A
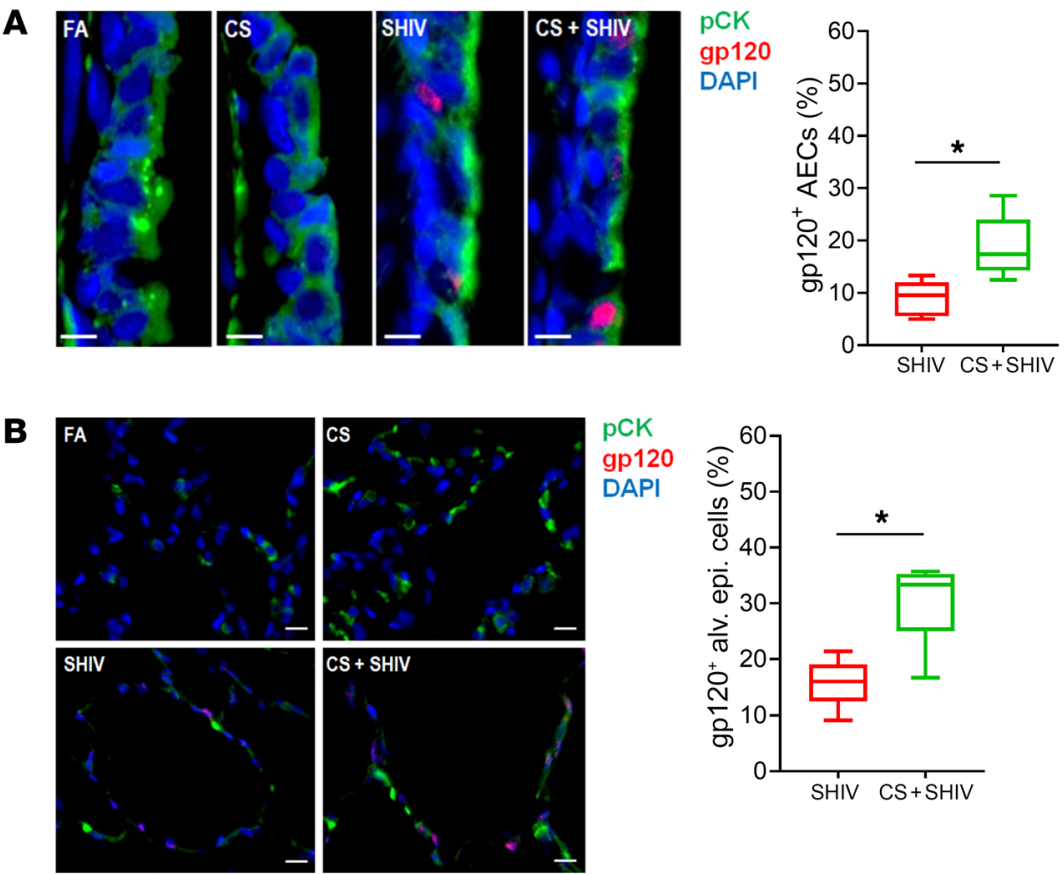

D
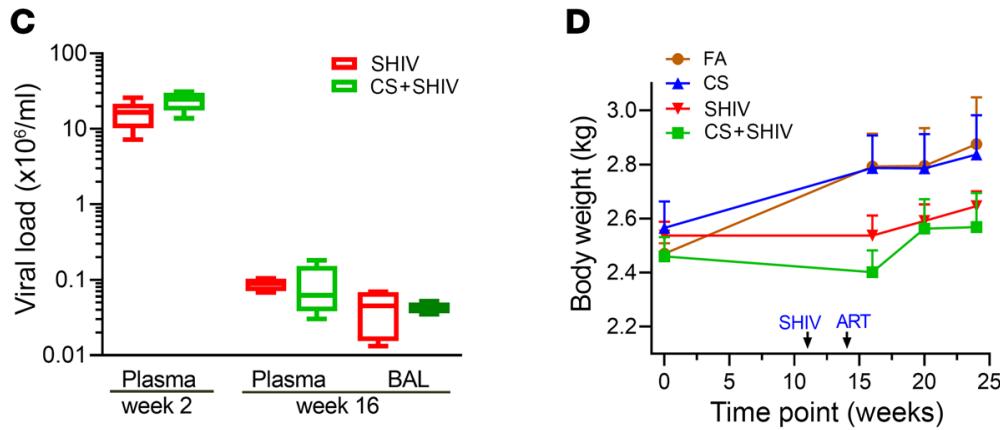

Figure 1. CS increases the number of gp120-immunopositive lung epithelial cells in SHIV-infected and cART-treated cynomolgus macaques. Formalin-fixed and paraffin-embedded (FFPE) lung tissue sections $(5 \mu \mathrm{m})$ from each group were immunostained for the epithelial cell marker pan-cytokeratin (pCK) and for HIV-gp120. (A) Representative micrographs of bronchial airway epithelial cells (AECs) showing pCK (green) and HIV-gp120 (red) immunopositivity along with the DAPI-stained nuclei (blue) from each group. Scale bars: $5 \mu \mathrm{m}$. Percentages of gp120+ AECs were quantified. (B) Micrographs of alveolar region showing pCK (green) and HIV-gp120 (red) immunopositivity from each group. The percentage of gp120 alveolar epithelial cells were quantified. Scale bars: 10 $\mu \mathrm{m}$. Data for $\mathbf{A}$ and $\mathbf{B}$ are mean $\pm \mathrm{SEM}, n=7 /$ group; ${ }^{*} P \leq$ 0.05 by Student's $t$ test. (C) Viral titers in the plasma and in the plasma and bronchoalveolar lavage (BAL) of SHIV and CS+SHIV-exposed animals at weeks 2 and 16 postinfection, respectively. (D) Body weights of macaques at the baseline and at indicated times during the experiment. Data are mean \pm SEM, $n=4-7 /$ group. tion. Titers didn't differ significantly $(P=0.088)$ between SHIV $\left(\sim 1.8 \times 10^{7}\right.$ copies $\left./ \mathrm{ml}\right)$ and CS+SHIV $\left(\sim 2.5 \times 10^{7}\right.$ copies $\left./ \mathrm{ml}\right)$ (Figure 1C). After 14 weeks of cART, the viral titers in SHIV and CS+SHIV dropped by more than $99.9 \%\left(\sim 8 \times 10^{3}\right.$ copies $\left./ \mathrm{ml}\right)$; viral titers in bronchoalveolar lavage (BAL) were also similar (Figure 1C). At 4 weeks postinfection, while the body weight of SHIV-infected and CS-exposed animals didn't differ $(P=0.09)$, CS+SHIV animals had significantly lower body weight than animals exposed to CS alone $(P=0.02)$. Following cART, CS+SHIV animals started regaining body weight and, at 3 weeks before sacrifice, their weights did not differ significantly from CS-exposed animals (Figure 1D). These results suggest: (a) cynomolgus macaques are extremely sensitive to $\mathrm{SHIV}_{89.6}$ infection; (b) the viral replication is sensitive to cART (viral loads decrease by $>99.9 \%$ ); (c) even after successful cART, SHIV infection induces HGECs in small airways and alveoli; and (d) the number of HGECs nearly doubles in animals chronically exposed to CS. However, presently we have no direct evidence suggesting that gp120 is synthesized by the gp120-immunoreactive epithelial cells. Nonetheless, the results support our earlier observation that the lungs of PLWH contain large numbers of HGECs (18) and it is likely that CS increases HGECs by either promoting HIV infection of epithelial cells or inhibiting the clearance of gp120+ epithelial cells.
Moreover, CS+SHIV affected the cellularity in BAL and the lung. Thus, compared with FA, BAL from CS+SHIV animals had significantly higher cell numbers (Supplemental Figure 2A) and neutrophil count (Supplemental Figure 2C). However, there was a significant decrease in BAL macrophages in CS+SHIV group (Supplemental Figure 2B) with no changes in BAL lymphocytes (Supplemental Figure 2D). Similarly, CS+SHIV animals exhibited markedly higher peribronchial, parenchymal, and perivascular lymphoid aggregates in the lung (Supplemental Figure 3). Thus, CS and SHIV infection synergistically affect lung pathology.

Smoking is a paramount risk factor for $\mathrm{CMH}$ that leads to $\mathrm{CB}$, decline in lung function, COPD, and asthma $(8,15,21-23)$. Although smoking is highly prevalent in PLWH, epidemiological evidence suggests HIV as an independent risk factor for CB and COPD (11, 12). MUC5AC and MUC5B are the 2 major secretory mucins in the airways that contribute to $\mathrm{CMH}$ and development of obstructive lung diseases $(17,22)$. When analyzed by quantitative reverse transcription PCR (qRT-PCR), compared with FA, MUC5AC mRNA was strongly upregulated by CS and SHIV and increased synergistically by CS+SHIV (Figure 2A, left panel); however, none of the treatments affected MUC5B levels (Figure 2A, right panel). Similarly, immunostaining of lung sections showed increased MUC5AC (Figure 2B) but not MUC5B (Figure 2C) in response to CS or SHIV. 
A
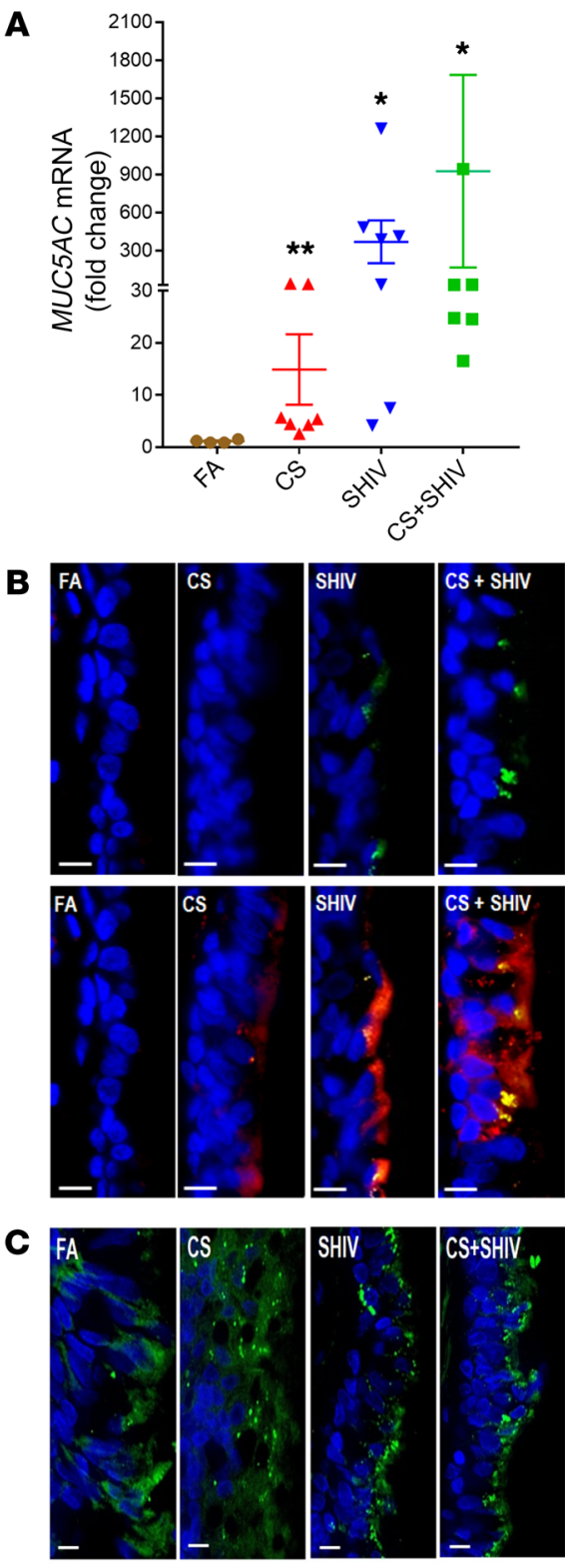

DAPI

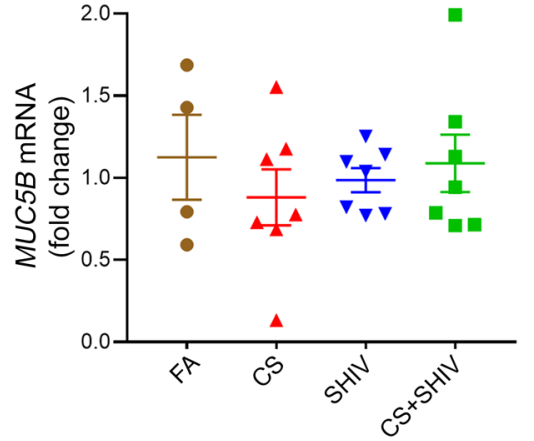

DAPI

gp120

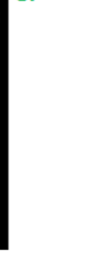

DAPI

gp120 MUC5AC
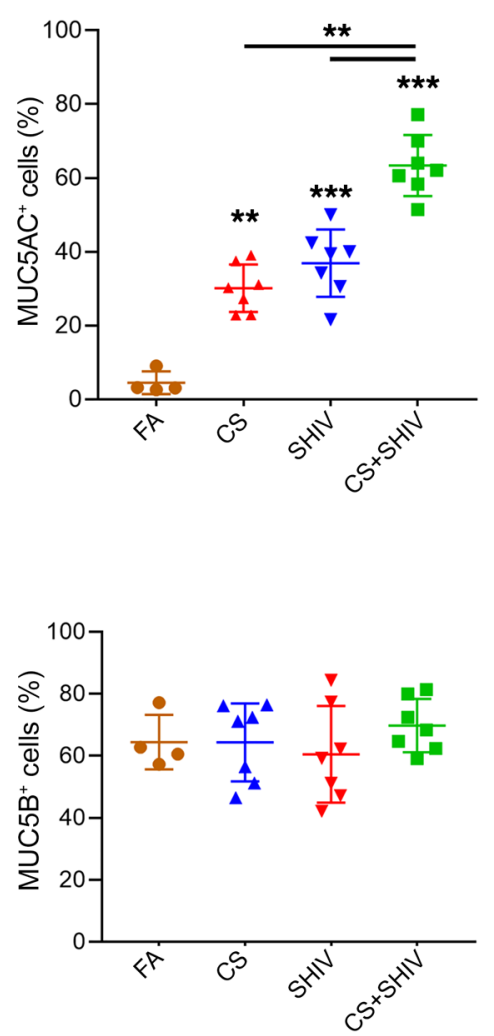

D

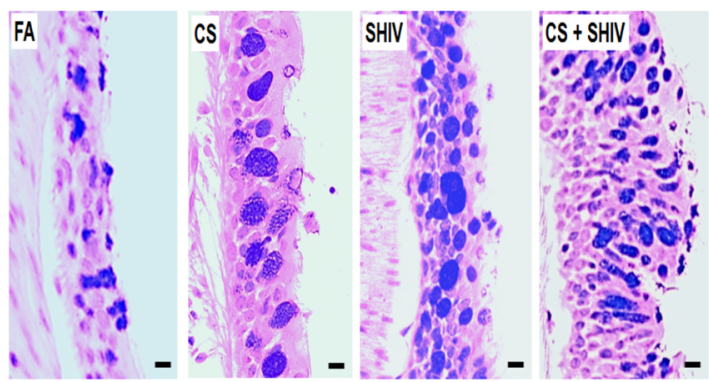

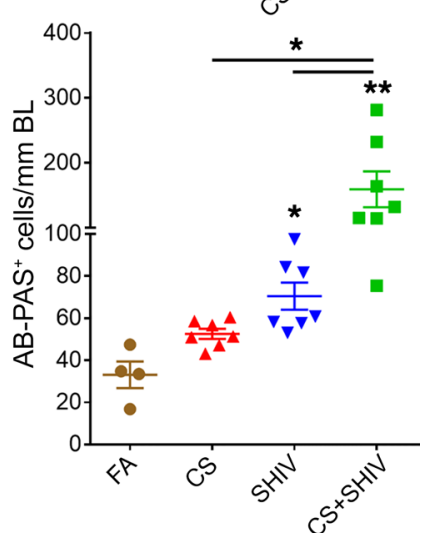

Figure 2. CS exposure and SHIV infection synergistically induce mucous cell hyperplasia and MUC5AC mucin expression. (A) Quantification of secretory mucin MUC5AC and MUC5B mRNA levels in each group. RNA was isolated from frozen lung tissues and assessed using real-time qRT-PCR. (B) Micrographs of small airways showing MUC5AC (red) and HIV-gp120 (green) immunopositivity from each group, and the percentage of $\mathrm{MUC5} \mathrm{AC}^{+}$epithelial cells. Scale bars: $5 \mu \mathrm{m}$. (C) Micrographs of small airways showing MUC5B (green) immunopositivity from each group, and the percentage of $\mathrm{MUC5B}^{+}$ epithelial cells. Scale bars: $5 \mu \mathrm{m}$. (D) Representative micrographs of small airways showing mucopolysaccharides stained with AB-PAS. Scale bars: $5 \mu \mathrm{m}$. AB-PAS+ cells were quantified per unit length $(\mathrm{mm})$ of basal lamina $(B L)$. Data are mean $\pm \mathrm{SEM}, n=4-7 /$ group, 1-way ANOVA; ${ }^{*} P \leq 0.05$; ${ }^{*} P<0.01$; ${ }^{* *} P<0.001$.

restricted to goblet cells and submucosal glands, respectively. MUC5B is critical for mucociliary clearance and antibacterial responses, whereas MUC5AC is overexpressed in allergic and obstructive lung diseases (24). In humans, MUC5AC and MUC5B are not regulated synchronously (24), thus, the effects of CS and/or SHIV appear localized primarily to goblet cells. Indeed, some MUC5 $\mathrm{AC}^{+}$cells are also HIV gp-120 immunoreactive (Figure 2B).

Differentiation of airway goblet cells requires interplay between several transcription factors. SAM-pointed domain-containing Ets-like factor (SPDEF) and forkhead box protein A3 (FOXA3) are selectively expressed by airway epithelial cells and stimulate mucus production, goblet cell hyperplasia/metaplasia, and proasthmatic cytokines like IL-13 and IL-4 (25). Using ELISA, we found that compared with FA, IL-13 levels are significantly upregulated in CS-exposed and SHIV-infected lungs $(P<0.01)$; CS+SHIV further increased the expression of IL-13 significantly (Supplemental Figure 4A). While FOXA3 and SPDEF are upregulated, the expression of FOXA2 that inhibits goblet cell and Th2 responses is downregulated in asthma and COPD patients (26-29). To assess the effects of CS \pm SHIV on these transcription factors, we determined the SPDEF, FOXA3, and FOXA2 mRNA levels by qRT-PCR (Supplemental Figure 4B). CS and SHIV independently increased SPDEF

Moreover, Alcian Blue-periodic acid Schiff reagent (AB-PAS) staining indicated that SHIV and CS independently promoted goblet cell hyperplasia and CS+SHIV dramatically exacerbated the response (Figure 2D). In airways, MUC5AC and MUC5B expression is and FOXA3 expression, which is increased further in CS+SHIV lungs. Interestingly, FOXA2 expression was independently reduced by CS and SHIV, and was essentially absent in CS+SHIV lungs. Thus, changes in the airway goblet cell differentiation, prompted by CS and SHIV, 
A
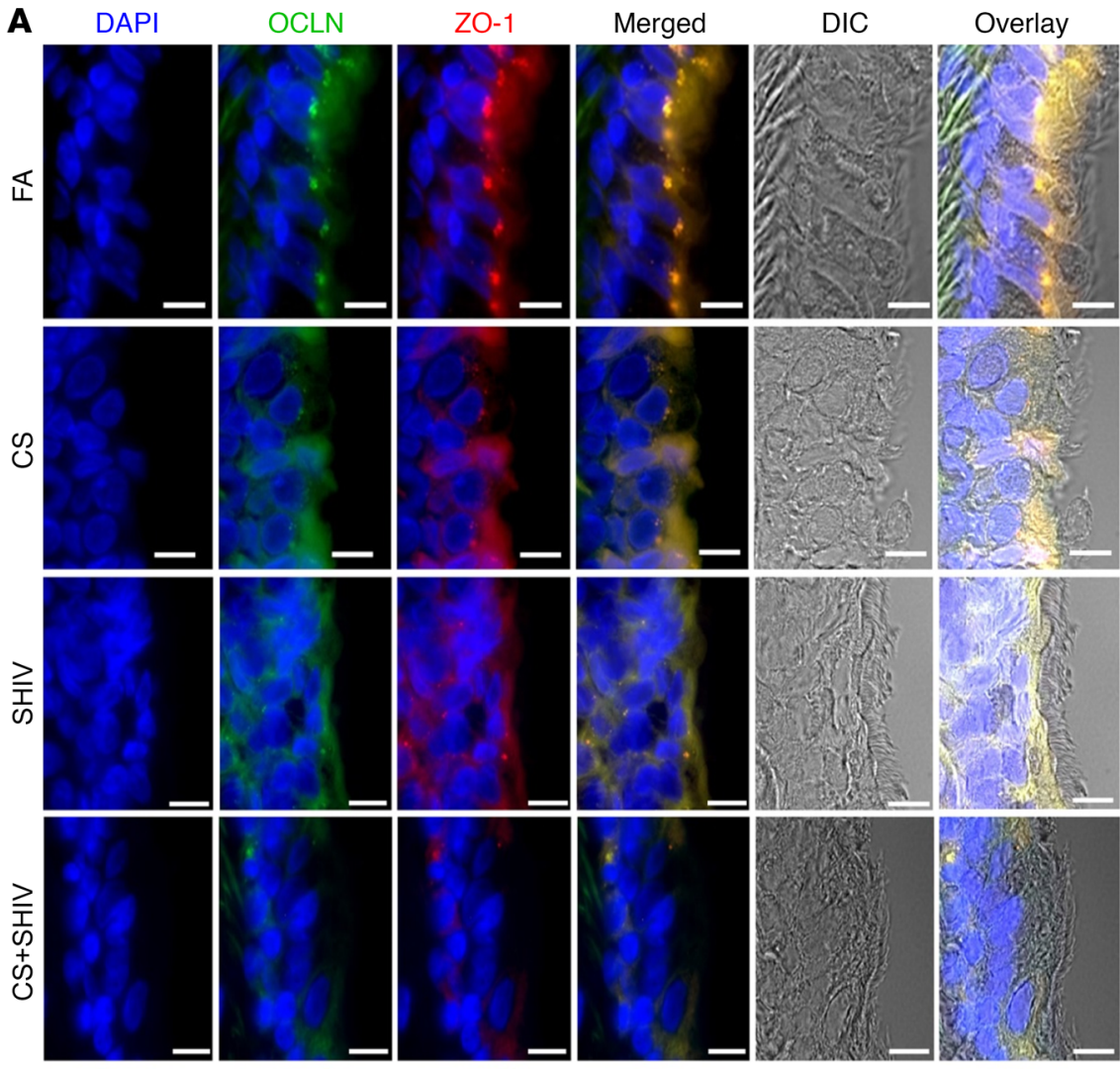

$\mathbf{B}$
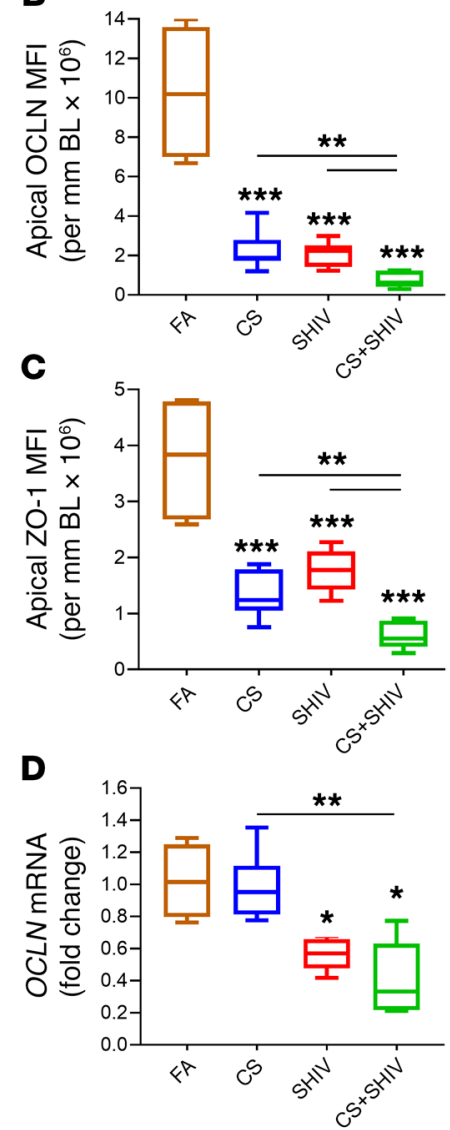

Figure 3. CS and SHIV independently and additively impair the lung epithelial tight junctions. (A) Representative micrographs of small airways showing tight junction proteins occludin (OCLN, green) and ZO-1 (red) along with the DIC (differential interference contrast) imaging. Scale bars: $5 \mu \mathrm{m}$. Quantification of apical OCLN (B) and ZO-1 (C) was assessed by measuring mean fluorescence intensity (MFI) per mm basal lamina (BL) in each group. (D) Quantification of OCLN mRNA levels in each group was determined by qRT-PCR. Data in B-D are mean \pm SEM, $n=4-7 /$ group, 1 -way ANOVA; $P \leq 0.05 ;{ }^{* *} P<0.01 ;{ }^{* * *} P<0.001$.

parallel the expression of transcription factors and cytokines that regulate mucous responses. Moreover, in human airways, IL-13 is a potent stimulator of MUC5AC but not MUC5B (24). Therefore, it is likely that SHIV infection and CS increases IL-13 expression, which promotes MUC5AC, CMH, and obstructive lung diseases. Although changes in IL-13, goblet cell hyperplasia, FOXA2, FOXA3, and SPDEF are compatible with proasthmatic responses, we have no direct evidence of asthma in these animals.

Given the extent of goblet cell hyperplasia in CS+SHIV airways, it was likely that the combination compromised the airway epithelial barrier that is dependent on the interaction of tight junction proteins such as occludin (OCLN) and cytosolic scaffolding proteins such as zonula occludins 1 (ZO-1) (30). Therefore, we stained airway sections for OCLN and ZO-1 and as shown in Figure 3A, the airway epithelium expressed double positive $\left(\mathrm{OCLN}^{+} \mathrm{ZO}-1^{+}\right)$cells in FA. However, CS and SHIV reduced the expression of OCLN and ZO-1 and the changes in the epithelial architecture were clearly evident by DIC (differential interference contrast images) and montage images of the immunohistographs (Figure 3A). Amounts of OCLN (Figure 3B) and ZO-1 (Figure 3C) were decreased independently by CS and SHIV, but the reduction was more prominent in CS+SHIV. Thus, CS and SHIV synergize to compromise the airway tight junctions that are critical in inflammatory lung diseases (31). Analysis of
OCLN mRNA by qRT-PCR (Figure 3D) indicated that, while SHIV downregulated OCLN expression, CS independently did not significantly affect the expression, and CS+SHIV was not significantly lower than SHIV alone. Therefore, CS does not affect the transcription of tight junction proteins but may affect their expression posttranslationally such as through phosphorylation (32).

Increased airflow limitation is an early sign of obstructive lung diseases such as COPD (33). Decreased forced expiratory volume at 1 second $\left(\mathrm{FEV}_{1}\right)$ reflects airflow obstruction in humans; however, in smaller animals (e.g., cynomolgus macaques), FEV at shorter times is more reproducible (34). We measured $\mathrm{FEV}_{0.1}$ and $\mathrm{FEV}_{0.15}$ at baseline and at 1 week prior to sacrifice. At baseline, $\mathrm{FEV}_{0.1}$ and $\mathrm{FEV}_{0.15}$ values were comparable (data not shown). However, while the $\mathrm{FEV}_{0.1}$ of FA, CS, and SHIV animals did not change over time - reflecting increased lung volumes in growing animals - the values were significantly lower in CS+SHIV (Figure 4A). Similar results were observed for $\mathrm{FEV}_{0.15}$ (data not shown). Thus CS+SHIV cooperate to induce airflow limitations compatible with the development of COPD-like changes.

$\mathrm{CB}$ and decreased $\mathrm{FEV}_{1}$ are directly related to increases in air wall thickness (35), and ultrasound (US) has been successfully used to visualize changes in upper airway structures (36). The epithelia of the cartilaginous airways are composed of mucosal glands, ciliated cells, and goblet cells, and goblet cells are the most common in the 
A

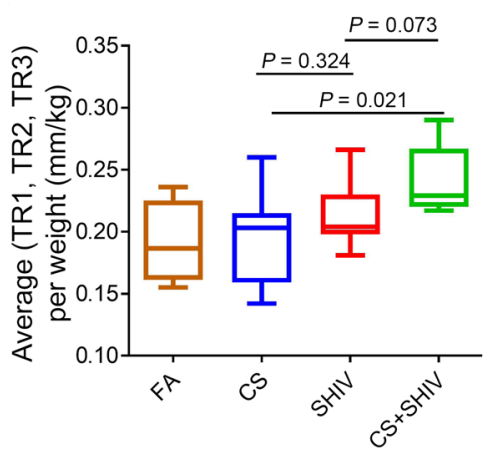

C

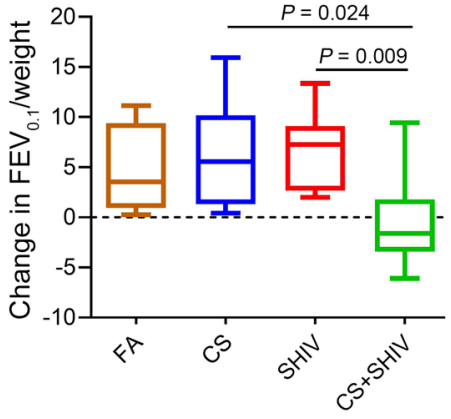

B

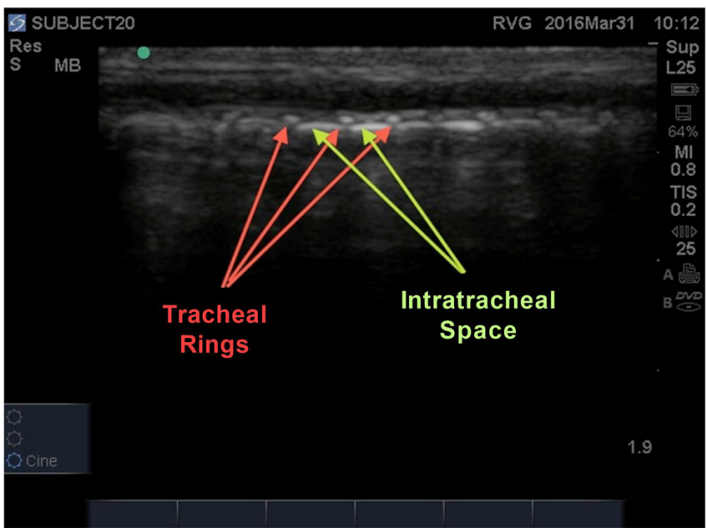

D

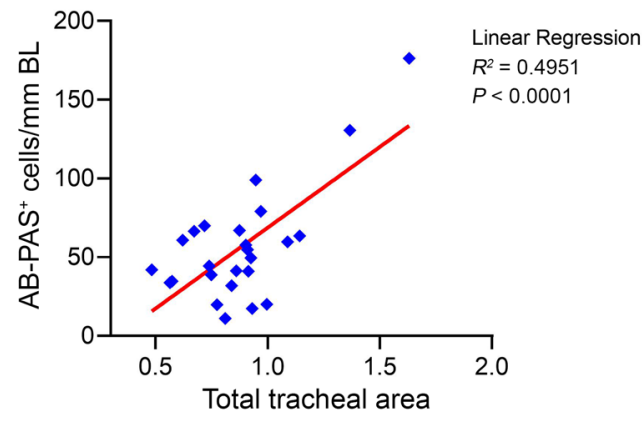

Figure 4. CS exposure and SHIV infection synergistically reduce lung function while augmenting upper airway wall thickness. (A) Changes in lung function parameters as determined by measuring $\mathrm{FEV}_{0.1}$ at baseline before the exposures and compared with the $F E V_{01}$ values at 1 week prior to sacrifice. (B) Representative ultrasonograph of upper airways showing the extrathoracic tracheal wall rings (TR1, TR2, and TR3) whose thickness was measured by ultrasound imaging. (C) Quantification of mean tracheal ring thickness among different treatment groups shows synergistic effect of CS and SHIV exposure. Data in $\mathbf{A}$ and $\mathbf{C}$ are mean $\pm \mathrm{SEM}, n=4-7 /$ group, 1-way ANOVA. (D) Linear regression analysis between airway mucous cell numbers/ $\mathrm{mm}$ basal lamina $(\mathrm{BL})$ and mean tracheal wall thickness (TR1 + TR2 + TR3) of all 25 animals. cephalad part of the trachea (37). Changes in extrathoracic proximal (subglottic) tracheal cartilage ring size have been reported in animal models and human adults with cystic fibrosis $(38,39)$. We sought to determine by US imaging whether SHIV and/or CS altered the thickness of the tracheal wall at the level of the tracheal rings (TRs). Longitudinal views of the sagittal plane that spanned from the lower margin of the larynx up to the trachea inferiorly were recorded at 27 weeks of CS exposure and offered the best anatomical details (Figure 4B). Mean thickness of tracheal rings (mTRs) encompassing the first, second, and third TRs and the mean size of intertracheal space between the tracheal rings TR1 and TR2 and TR2 and TR3 were measured and normalized to body weights. Results suggested that none of the treatments caused significant changes in the size of tracheal spaces (data not shown). However, mTR of CS+SHIV was significantly higher than that of CS alone $(P=0.02)$ and was not significantly higher than that of SHIV alone $(P=0.07)$ (Figure $4 \mathrm{C}$ ). These data suggest that CS+SHIV synergize to stimulate structural changes in the airways that are visualized by US in cynomolgus macaques. Moreover, linear regression analysis suggested a significant correlation between the number of airway mucous cells and the mean tracheal wall thickness (Figure 4D). COPD in HIV-infected patients is underdiagnosed (40) and, although COPD is a progressive disease, early stage interventions may reduce the rate of lung function decline and improve mortality (41). Although we have not done any human testing, it is possible that US of tracheal rings has the potential to monitor CMH/COPD-like changes in PLWH.

Persistent bacterial (42) and viral (43) lung infections have been proposed as COPD inducers. Adenovirus EA1 expression was correlated to accelerated COPD/emphysema in smokers but not in nonsmokers (44).However, to our knowledge, there are no published reports directly linking viral infection alone to the development of $\mathrm{CB} / \mathrm{COPD}$.
Differing lifestyles, lung infections, and nearly ubiquitous smoking in PLWH make it difficult to analyze the respiratory effects of HIV; however, epidemiological studies suggest HIV-infection as an independent risk factor for obstructive lung diseases $(3,45,46)$. This study provides clear experimental evidence of $\mathrm{HIV}$ as an independent risk factor for development of CB- and COPD-like changes in the lung. In addition, changes in the epithelial barrier and upper airway structures underscore the inference that the lung undergoes COPD-like changes in response to CS or SHIV-infection that are accelerated in animals exposed to CS+SHIV. Furthermore, the results suggest that the lung may be an important HIV reservoir and chronic HIV infection, even after cART, promotes obstructive changes in the lung. If so, PLWH who are also smokers are at much higher risk of developing noninfectious chronic lung conditions than PLWH who are nonsmokers.

\section{Methods}

For more details, see Supplemental Methods.

Statistics. Statistical methods are described in Supplemental Methods. Briefly, grouped results were expressed as mean \pm SEM and $P$ values less than or equal to 0.05 were considered significant.

Study approval. The animals were housed at the Primate Facility of Lovelace Respiratory Research Institute, Albuquerque, NM, in accordance with the Guidelines from the Association for the Assessment and Accreditation for Laboratory Animal Care International. All experimental protocols carried out on these macaques were approved by the Institutional Animal Care and Use Committee.

\section{Author contributions}

HSC performed the sample analysis, analyzed the data, and wrote the manuscript. RVG, CR, KR, and NM performed the study and analyzed the data. SPS, SSH, and SNB performed the sample analysis and 
analyzed the data. EB, SC, FV, and SB analyzed the data. MS designed the studies, analyzed the data, and wrote the manuscript. MCVG and JA read the ultrasound. AS, VE, VR, and HA read pathology slides. MN designed the HIV experiments. All authors reviewed the manuscript.

\section{Acknowledgments}

We thank the staff and veterinarians of the Inhalation Core facility and Julie Hutt (Histology Core) of Lovelace Respiratory Research
Institute for helping us carry out these studies. This work was supported by NIH grants R01HL125000 (to MS), R21AI117560 (to HSC), R01DA040537 and R01DA03783 (to MN), and by an American Lung Association grant RG306208 (to HSC).

Address correspondence to: Mohan Sopori, Lovelace Respiratory Research Institute, 2425 Ridgecrest Drive SE, Albuquerque, New Mexico 87108, USA. Phone:505.348.9440; Email: msopori@lrri.org.
1. Niaura R, Shadel WG, Morrow K, Tashima K, Flanigan T, Abrams DB. Human immunodeficiency virus infection, AIDS, and smoking cessation: the time is now. Clin Infect Dis. 2000;31(3):808-812.

2. George MP, Kannass M, Huang L, Sciurba FC, Morris A. Respiratory symptoms and airway obstruction in HIV-infected subjects in the HAART era. PLoS One. 2009;4(7):e6328.

3. Crothers K, et al. HIV infection and risk for incident pulmonary diseases in the combination antiretroviral therapy era. Am J Respir Crit Care Med. 2011;183(3):388-395.

4. Foster SB, et al. Increased incidence of asthma in HIV-infected children treated with highly active antiretroviral therapy in the National Institutes of Health Women and Infants Transmission Study. J Allergy Clin Immunol. 2008;122(1):159-165.

5. Gingo MR, Morris A, Crothers K. Human immunodeficiency virus-associated obstructive lung diseases. Clin Chest Med. 2013;34(2):273-282.

6. Drummond MB, Kunisaki KM, Huang L. Obstructive lung diseases in HIV: a clinical review and identification of key future research needs. Semin Respir Crit Care Med. 2016;37(2):277-288.

7. Forey BA, Thornton AJ, Lee PN. Systematic review with meta-analysis of the epidemiological evidence relating smoking to COPD, chronic bronchitis and emphysema. BMC Pulm Med. 2011;11:36.

8. McLeish AC, Zvolensky MJ. Asthma and cigarette smoking: a review of the empirical literature. JAsthma. 2010;47(4):345-361.

9. Kwong J, Bouchard-Miller K. Smoking cessation for persons living with HIV: a review of currently available interventions. J Assoc Nurses AIDS Care. 2010;21(1):3-10.

10. Browning KK, Wewers ME, Ferketich AK, Diaz P. Tobacco use and cessation in HIV-infected individuals. Clin Chest Med. 2013;34(2):181-190.

11. Diaz PT, et al. Increased susceptibility to pulmonary emphysema among HIV-seropositive smokers. Ann Intern Med. 2000;132(5):369-372.

12. Crothers $\mathrm{K}$, et al. Increased COPD among HIV-positive compared to HIV-negative veterans. Chest. 2006;130(5):1326-1333.

13. Donaldson GC, Seemungal TA, Bhowmik A, Wedzicha JA. Relationship between exacerbation frequency and lung function decline in chronic obstructive pulmonary disease. Thorax. 2002;57(10):847-852.

14. Wedzicha JA. Airway mucins in chronic obstructive pulmonary disease. N Engl J Med. 2017;377(10):986-987.

15. Kim V, Criner GJ. Chronic bronchitis and chronic obstructive pulmonary disease. Am J Respir Crit Care Med. 2013;187(3):228-237.
16. Fahy JV, Dickey BF. Airway mucus function and dysfunction. NEngl JMed. 2010;363(23):2233-2247.

17. Kesimer M, et al. Airway mucin concentration as a marker of chronic bronchitis. $N$ Engl J Med. 2017;377(10):911-922.

18. Gundavarapu S, et al. Role of nicotinic receptors and acetylcholine in mucous cell metaplasia, hyperplasia, and airway mucus formation in vitro and in vivo. J Allergy Clin Immunol. 2012;130(3):770-780.e11.

19. Brune KA, et al. HIV impairs lung epithelial integrity and enters the epithelium to promote chronic lung inflammation. PLoS One. 2016;11(3):e0149679.

20. Chinnapaiyan S, et al. HIV infects bronchial epithelium and suppresses components of the mucociliary clearance apparatus. PLoS One. 2017;12(1):e0169161.

21. Vestbo J. Epidemiological studies in mucus hypersecretion. Novartis Found Symp. 2002;248:3-12; discussion 12.

22. Ha EV, Rogers DF. Novel therapies to inhibit mucus synthesis and secretion in airway hypersecretory diseases. Pharmacology. 2016;97(1-2):84-100.

23. Allinson JP, Hardy R, Donaldson GC, Shaheen SO, Kuh D, Wedzicha JA. The presence of chronic mucus hypersecretion across adult life in relation to chronic obstructive pulmonary disease development. Am J Respir Crit Care Med. 2016;193(6):662-672.

24. Bonser LR, Erle DJ. Airway mucus and asthma: the role of MUC5AC and MUC5B. JClin Med. 2017;6(12):E112.

25. Rajavelu P, Chen G, Xu Y, Kitzmiller JA, Korfhagen TR, Whitsett JA. Airway epithelial SPDEF integrates goblet cell differentiation and pulmonary Th2 inflammation. JClin Invest. 2015;125(5):2021-2031.

26. Chen G, et al. Foxa3 induces goblet cell metaplasia and inhibits innate antiviral immunity. Am J Respir Crit Care Med. 2014;189(3):301-313.

27. Tang X, et al. Foxa2 regulates leukotrienes to inhibit Th2-mediated pulmonary inflammation. Am J Respir Cell Mol Biol. 2013;49(6):960-970.

28. Park SW, et al. Distinct roles of FOXA2 and FOXA3 in allergic airway disease and asthma. Am J Respir Crit Care Med. 2009;180(7):603-610.

29. Song J, et al. Aberrant DNA methylation and expression of SPDEF and FOXA2 in airway epithelium of patients with COPD. Clin Epigenetics. 2017;9:42.

30. Mitchell LA, et al. Junctional adhesion molecule A promotes epithelial tight junction assembly to augment lung barrier function. Am J Pathol. 2015;185(2):372-386.

31. Wittekindt $\mathrm{OH}$. Tight junctions in pulmonary epithelia during lung inflammation. Pflugers Arch. 2017;469(1):135-147.

32. Raleigh DR, et al. Occludin S408 phosphorylation regulates tight junction protein interactions and barrier function. JCell Biol. 2011;193(3):565-582.

33. Hogg JC, et al. The nature of small-airway obstruction in chronic obstructive pulmonary disease. N Engl JMed.2004;350(26):2645-2653.

34. Kling HM, Shipley TW, Guyach S, Tarantelli R, Morris A, Norris KA. Trimethoprim-sulfamethoxazole treatment does not reverse obstructive pulmonary changes in pneumocystis-colonized nonhuman primates with SHIV infection. J Acquir Immune Defic Syndr. 2014;65(4):381-389.

35. Kim V, et al. Clinical and computed tomographic predictors of chronic bronchitis in COPD: a cross sectional analysis of the COPDGene study. Respir Res. 2014;15:52.

36. Lun HM, Zhu SY, Liu RC, Gong JG, Liu YL. Investigation of the upper airway anatomy with ultrasound. Ultrasound Q. 2016;32(1):86-92.

37. Ohtsuka R, Doi K, Itagaki S. Histological characteristics of respiratory system in Brown Norway rat. Exp Anim. 1997;46(2):127-133.

38. Bonvin $\mathrm{E}$, et al. Congenital tracheal malformation in cystic fibrosis transmembrane conductance regulator-deficient mice. J Physiol (Lond). 2008;586(13):3231-3243.

39. Diwakar A, et al. Sonographic evidence of abnormal tracheal cartilage ring structure in cystic fibrosis. Laryngoscope. 2015;125(10):2398-2404

40. Risso K, et al. COPD in HIV-infected patients: CD4 cell count highly correlated. PLoS One. 2017;12(1):e0169359.

41. Tashkin DP, Celli B, Kesten S, Lystig T, Mehra S, Decramer M. Long-term efficacy of tiotropium in relation to smoking status in the UPLIFT trial. Eur Respir J. 2010;35(2):287-294.

42. Ishak A, Everard ML. Persistent and recurrent bacterial bronchitis-a paradigm shift in our understanding of chronic respiratory disease. Front Pediatr. 2017;5:19.

43. Matsumoto K, Inoue H. Viral infections in asthma and COPD. Respir Investig. 2014;52(2):92-100.

44. Retamales I, et al. Amplification of inflammation in emphysema and its association with latent adenoviral infection. Am J Respir Crit Care Med. 2001;164(3):469-473.

45. Gingo MR, et al. Pulmonary symptoms and diagnoses are associated with HIV in the MACS and WIHS cohorts. BMC Pulm Med. 2014;14:75.

46. Nakamura $\mathrm{H}$, et al. The prevalence of airway obstruction among Japanese HIV-positive male patients compared with general population; a case-control study of single center analysis. J Infect Chemother. 2014;20(6):361-364. 